mass, the patient's mean arterial pressure increased to $140 \mathrm{~mm} \mathrm{Hg}$. The aorta was crossclamped, and the heart was arrested and emptied. The mass was resected, and the defect in the right atrium was closed with a pericardial patch. The patient was weaned easily from cardiopulmonary bypass. Norepinepherine levels taken from a coronary sinus catheter before and after resection went from 859 to $249 \mathrm{pg} / \mathrm{mL}$.

The patient's course was uncomplicated, and he was discharged on postoperative day 5 . His only antihypertensive medication at discharge was an angiotensin-converting enzyme inhibitor. Histopathologic examination demonstrated a $5 \mathrm{~cm} \times 5 \mathrm{~cm} \times 3 \mathrm{~cm}$ PCT.

\section{Discussion}

PCTs are tumors of the sympathetic nervous system arising from chromaffin cells. In adults the majority of these masses are located in the adrenal medulla, where chromaffin cells are concentrated. Ten percent occur in extra-adrenal locations, with the organ of Zuckerkandl being the most common. Chromaffin cells can also be found in the walls of blood vessels, along the aorta, and in the heart, prostate, and ovaries. PCTs produce large amounts of catecholamines, particularly norepinephrine. These tumors occur in less than $0.1 \%$ of hypertensive patients.

In a review of 30 cases, ${ }^{1}$ the most common presentation of PCT was hypertension, and dyspnea accounted for only 3 cases. Although our patient was hypertensive at the time of presentation, it was his symptoms of heart failure that prompted the echocardiogram. On magnetic resonance imaging, its signal characteristics suggested a vascular lesion, such as angiosarcoma, hemangioma, or PCT.,3

Coronary angiography is recommended before resection. At least one case reported reimplantation of a coronary artery. ${ }^{1}$
Most cardiac PCTs are densely adherent to underlying tissues. We and others recommend cardiopulmonary bypass and aortic crossclamping for resection. ${ }^{4}$ Manipulation of the tumor can cause marked hypertension, even if preoperative $\alpha$ - and $\beta$-blockade is performed. Wilson and colleagues ${ }^{5}$ described systolic blood pressures of greater than $300 \mathrm{~mm} \mathrm{Hg}$ when a PCT was resected through a right thoracotomy with no bypass. Four deaths have been described in a review of 25 patients, all resulting from massive hemorrhage.

\section{Conclusion}

Cardiac PCTs represent interesting diagnostic and treatment dilemmas. Although rare, they can be treated for cure in more than $90 \%$ of cases. Magnetic resonance imaging is the noninvasive test of choice. Cardiac catheterization is recommended and aids in surgical management. These authors advocate excision with cardiopulmonary bypass and cardioplegic arrest.

\section{References}

1. Jebara VA, Uva MS, Farge A, et al. Cardiac pheochromocytomas. Ann Thorac Surg. 1992;53:356-61.

2. Grebenc ML, Rosado de Christenson ML, Burke AP, Green CE, Galvin JR. Primary cardiac and pericardial neoplasms: radiologic-pathologic correlation. Radiographics. 2000;20:1073-103.

3. Araoz PA, Mulvagh SL, Tazelaar HD, Julsrud PR, Breen JF. CT and MR imaging of benign primary cardiac neoplasms with echocardiographic correlation. Radiographics. 2000;20:1303-19.

4. Orringer MB, Sisson JC, Glazer G, Shapiro B, Francis I, Behrendt DM, et al. Surgical treatment of cardiac pheochromocytomas. J Thorac Cardiovasc Surg. 1985;89:753-7.

5. Wilson AC, Bennett RC, Niall JF, et al. An unusual case of intrathoracic pheochromocytoma. Aust N Z J Surg. 1974;44:27-32.

\title{
Internal thoracic artery injury after transvenous pacemaker implantation
}

Alan P. Kypson, MD, ${ }^{a}$ David W. Frazier, MD, ${ }^{b}$ and Jon F. Moran, MD, ${ }^{a}$ Greenville, NC

I njuries associated with transvenous insertion of pacemaker leads occur infrequently. Common complications include pneumothorax-hemothorax, subclavian vein and artery injury, and myocardial perforation. ${ }^{1}$ We describe, for the first time, a laceration of the left internal thoracic artery (LITA) diagnosed 2 days after pacemaker implantation.

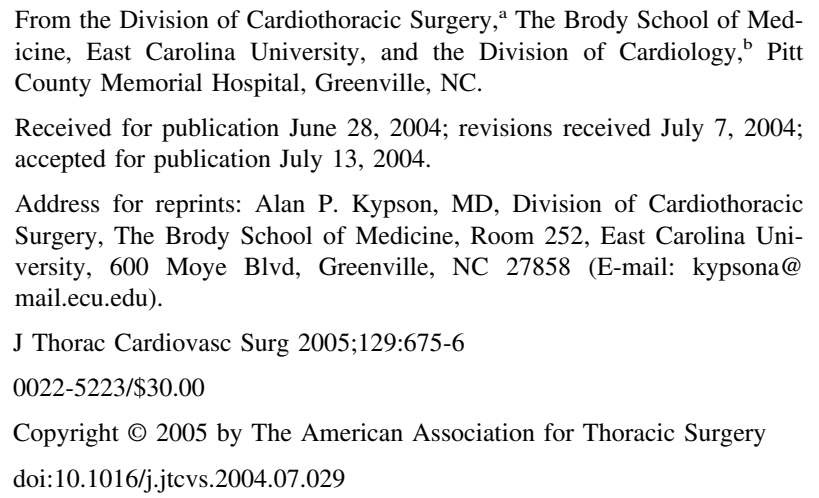

\section{Clinical Summary}

A 71-year-old man with a medical history significant for sick sinus syndrome and atrioventricular nodal conduction system disease underwent an insertion of a dual-chambered pacemaker. Screw-in pacing leads were placed transvenously and fixed in the right atrial appendage and right midventricular septum. The generator was implanted in a subcutaneous pocket in the left side of the chest. Pacing thresholds were adequate. Chest radiography revealed both leads to be in good position. The patient was discharged home the next day.

Thirty-six hours later, the patient presented in respiratory distress with left-sided chest pain. Initial systolic blood pressure was $70 \mathrm{~mm} \mathrm{Hg}$. Hemoglobin was $10.1 \mathrm{~g} / \mathrm{dL}$. Cardiac enzymes were within normal limits. Chest radiography showed opacification of the left hemithorax. Echocardiography showed no pericardial effusion. Computed tomography demonstrated a left hemothorax with extrusion of the pacing lead into the left hemithorax (Figure 1).

Tube thoracostomy was performed, and $1.5 \mathrm{~L}$ of blood was drained. Subsequently, the patient bled about $400 \mathrm{~mL} / \mathrm{h}$ and therefore underwent emergency thoracotomy. An actively bleeding 


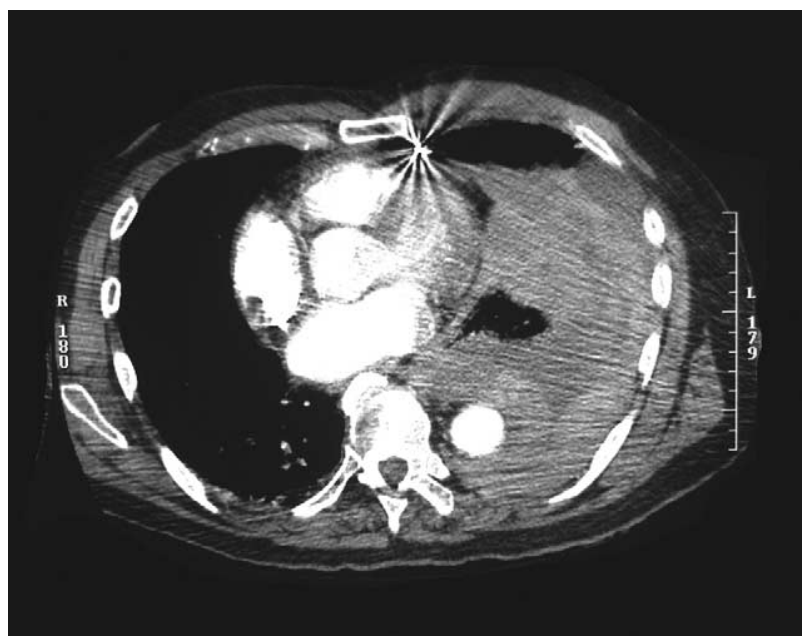

Figure 1. Cardiac perforation with a pacing lead into the left thoracic cavity, as demonstrated by means of computed tomographic scanning.

lacerated LITA was identified and suture ligated. The ventricular pacing lead perforated the pericardium with its tip 2 to $3 \mathrm{~cm}$ from the LITA. The pericardium was opened, and the lead was cut at the epicardial level, allowing the proximal portion to retract into the heart. The puncture site was observed, and after digital pressure, there was no bleeding. The pericardium was left open, and the incision was closed in standard fashion. Postoperatively, the patient underwent revision of his pacemaker system, with removal of the remainder of the right ventricular lead followed by insertion of a new one.

\section{Conclusion}

Pacemakers and implantable cardioverter-defibrillators are being placed at an increasing frequency. The ease of implantation has allowed this procedure to be performed on an outpatient basis. Nevertheless, it is a procedure that carries its own set of complications. Recently, a report showed a pacemaker implantation complication rate of $4 \%$ to $5 \% .^{2}$ The incidence of implant complications has been shown to be similar between single- and dual-chamber pacemakers, as well as dependent on the experience of the operator. ${ }^{3,4}$

Implant complications can be classified as pocket related, venous access related, and lead related. Pocket-related complications usually consist of hematomas that occasionally require evacuation. Long-term complications include skin erosion if the pocket is too tight. Attempts at subclavian venous access can cause complications, such as pneumothorax, hemothorax, and air embolism.
Pneumothorax is operator dependent and varies with the degree of difficulty of the puncture. This complication can be virtually eliminated by use of the cephalic cutdown technique. Hemothorax usually results from trauma to the great vessels. This can be minimized by using the needle technique (in and out rather than side to side) and by confirming the path of the guide wire under fluoroscopy.

Lead-related complications can often be the most serious. The lead might perforate any of the great veins, atria, or ventricles during the implant procedure. Trauma to the great veins outside the pericardial reflection can cause bleeding into the mediastinum. Usually, these injuries are seen with lead-extraction procedures. Lead perforation through the heart itself typically occurs with lead manipulation or when screwing in a lead. Usually, withdrawal of the lead stops the bleeding. However, a life-threatening emergency can arise if the bleeding continues progressing to cardiac tamponade, which requires immediate pericardiocentesis, followed by surgical intervention.

In this case there was no documented hemopericardium. Rather, lead perforation was suggested by the computed tomographic scan and confirmed at the time of the operation. Most likely, the lead was placed through the pericardium at the time of implantation and lodged into the LITA. Thereafter, the lead became detached, resulting in active bleeding.

In conclusion, complications of pacemaker insertion can masquerade as other less serious entities with dyspnea or chest pain, especially if time has elapsed since the procedure. Screening studies help guide the patient's management. It is important to keep in mind the complication of cardiac perforation. In this case the perforation itself was not life-threatening but rather the laceration of the LITA. Although the subclavian vessels are most commonly injured during pacemaker implantation, we have documented, for the first time, an injury to another major blood vessel within the thoracic cavity. This injury should be kept in the differential diagnosis when examining patients who have deviated from the normal postprocedural course.

\section{References}

1. Pavia S, Wilkoff B. The management of surgical complications of pacemaker and implantable cardioverter-defibrillators. Curr Opin Cardiol. 2001;16:66-71.

2. Tobin K, Stewart J, Westveer D, Frumin H. Acute complications of permanent pacemaker implantation: their financial implication and relation to volume and experience. Am J Cardiol. 2000;85:774-6.

3. Mueller X, Hossein S, Kappenberger L. Complications after single versus dual chamber pacemaker implantation. PACE. 1990;13:711-4.

4. Parsonnet V, Bernstein A, Lindsay B. Pacemaker-implantation complication rates: an analysis of some contributing factors. J Am Coll Cardiol. 1989;13:917-21. 